\title{
ON A DECOMPOSITION OF TRUE CYCLIC ELEMENTS*
}

\author{
BY \\ DICK WICK HALI $\dagger$ \\ INTRODUCTION \\ Part I. DeVElopment OF THE THEORY \\ Part II. Applications \\ PART III. EXAMPLES
}

\section{INTRODUCTION}

The study of locally connected continua has been greatly simplified by the introduction of the cyclic element concept of G. T. Whyburn. $\ddagger$ By definition, a cyclic element of a locally connected continuum $M$ is any subset $E$ of $M$ satisfying some one of the following conditions: (1) $E$ is a cut point of $M$; (2) $E$ is an end point of $M$; (3) $E$ is any nondegenerate subcontinuum of $M$ containing no cut point of itself which is saturated $\S$ in $M$ with respect to this property. Every cyclic element of type (3) is called a true cyclic element.

Along these lines much light has been shed upon the knotty problem of the structure of a locally connected continuum $M$ by considering $M$ as the sum of its cyclic elements and then deducing properties of $M$ from the properties of cyclic elements and the known relationships which exist between them.

The richness of the results in this direction has led to the hope that some still finer decomposition of locally connected continua may be possible. One such finer decomposition, based on combinatorial concepts, is given by the cyclic elements of higher order. $\|$ It is to the problem of obtaining a decomposition in the set theoretic direction that the present paper is devoted. We begin with a true cyclic element $C$ as our space and attempt to decompose it into subsets having desirable properties. The decomposition of the space into its cyclic elements then gives the decomposition we want.

This paper is but a first step towards the ultimate goal of an extensive structure theory of true cyclic elements. It explores only the most fundamen-

\footnotetext{
* Presented to the Society, April 9, 1938, and October 29, 1938; received by the editors June 26, 1939, and, in revised form, December 19, 1939.

† This paper was completed while the author was a National Research Fellow at the University of Pennsylvania.

$\ddagger$ See Kuratowski and Whyburn, Fundamenta Mathematicae, vol. 16 (1930), pp. 305-331. In this article an extensive bibliography may be found.

$\S$ A subset $E$ of $M$ is said to be saturated in $M$ with respect to a property $P$ provided $E$ has property $P$ and no subset of $M$ containing $E$ but distinct from $E$ has this property.

|l See G. T. Whyburn, American Journal of Mathematics, vol. 56 (1934), pp. 133-146.
} 
tal properties of our new elements, and the most elementary relationships existing between pairs of such sets and between such sets and their complements. It leaves for future study such considerations as the following:

(1) The possible existence of analogues of simple cyclic chains.

(2) The possible existence of analogues of $A$-sets.

(3) The study of the hyperspace formed when each secondary element is considered as a single point.

The author wishes to state that he benefited greatly from conversations with Dr. G. E. Schweigert during the earlier parts of the work.

\section{Part I. Development of the theory}

Let $C$ be a cyclicly connected continuous curve which is not a simple closed curve.

Lemma 1. Every free arc* of $C$ is a subset of a maximal free arc of $C$.

Proof. Let $\alpha$ be a free arc and $a$ be an interior point of $\alpha$. Let $J$ be a simple closed curve containing $a$. Obviously $J \supset \alpha$. On each of the two arcs of $J$ from $a$ let $x$ and $y$ be the first point of the closed set $\overline{C-J}$. Since $C \neq J, x$ and $y$ exist. Further $x \neq y$, for otherwise this single point would be a cut point of $C$. Then $x a y$ is a free arc containing $\alpha$ and maximal.

We now define the following terms:

(a) A 2-set consists of any pair of points of $C$ which separate $C$ and do not lie together on the same free $\operatorname{arc}$ of $C$.

(b) A 2-point of $C$ is any point $h$ belonging to a 2-set of $C$.

(c) Two points $a$ and $b$ of $C$ are biconjugate if no 2 -set separates $C$ between $a$ and $b$.

(d) By a secondary element of $C$ we shall mean any subset $E$ of $C$ satisfying some one of the following conditions:

I. $E$ consists of a non 2-point $a$ of $C$ together with all points $b$ of $C$ biconjugate to $a$, and there is at least one such point $b$. In this case $E$ is called a true secondary element. A true secondary element is said to be nondegenerate provided that it contains at least one nondegenerate component.

II. $E$ is a non 2-point $a$ such that no point $b$ of $C$ is biconjugate to $a$. In this case $a$ is called a 2-end point.

III. $E$ is a maximal free arc of $C$ containing at least one 2-point in its interior.

IV. $E$ is a 2 -set.

* An arc $a x b$ of $C$ is called a free arc of $C$ provided the interior of $a x b$, which we denote by $\langle a x b\rangle$, is an open subset of $C$. We shall denote the sets $a x b-a$ and $a x b-b$ by $\langle a x b$ and $a x b\rangle$, respectively. An $\operatorname{arc} a x b$ is said to span a point set $M$ provided $a x b \cdot M=a+b$. This lemma is an unpublished result of G. C. Watson. The proof here given was suggested by the referee. 
The following theorems are easily established:

THEOREM I. (a) The space is the sum of its secondary elements, in fact every point $p$ of $C$ is contained in a secondary element of type I, II, or IV.

(b) Every secondary element is a closed set.

(c) Every two points of a true secondary element are biconjugate.

(d) Every true secondary element is independent of the non 2-point defining it, that is, it may equally well be defined by any non 2-point which it contains.

(e) Every non 2-point belongs to at most one true secondary element.

(f) The product of two true secondary elements is vacuous, a 2-point, or a 2-set.

(g) If $A$ be a free arc of $C$ with end points $a$ and $b$ containing at least one 2-point $r$ in its interior, then every 2-set $K$ of $C$ which contains $r$ separates $C$ between $a$ and $b$.

(h) If $A$ be a free arc of $C$, then a necessary and sufficient condition that $A$ contain a pair of biconjugate points is that every interior point of $A$ be a non 2-point.

(i) If $E$ be a true secondary element, then every interior point of every free arc of $E$ is a non 2-point.

(j) If $E$ be a secondary element of type III, then every interior point of $E$ is a 2-point and $E$ contains no biconjugate pair of points.

(k) If $E$ be a true secondary element containing $b$ and $c$, then $E$ contains every free arc joining $b$ and $c$ in $C$.

THEOREM II. Let $E$ be a true secondary element and $K$ any component of $C-E$. Then

(a) $F(K)=\bar{K}-K$ consists of exactly two points, $r$ and $s$;

(b) both $r$ and $s$ are local separating points of $C$ of order greater than 2;

(c) either $r$ or $s$ is a 2-point.

Proof. (a) Since $C$ is cyclic, $F(K)$ contains at least two points $x, y$. Let $z$ be any third point of $F(K)$. Let $U$ and $W$ be regions containing $x$ and $z$, respectively, and having disjoint closures neither of which contains the point $y$. Let $x^{\prime}, z^{\prime}$ be arbitrary points in $K U, K W$. There exists a simple $\operatorname{arc} x^{\prime} z^{\prime}$ in $K$; hence $x^{\prime} z^{\prime}$ does not contain $y$. Let $V$ be a region in $C$, containing $y$, such that $\bar{V}$ is disjoint with each of the three sets $\bar{U}, \bar{W}$, and $x^{\prime} y^{\prime}$. Let $x^{\prime} x, z^{\prime} z$ be simple arcs in $U, W$, respectively, and $x^{\prime \prime}, z^{\prime \prime}$ the first points of $E$ on these arcs (Theorem I(b)). It follows easily that there exists a simple arc $x^{\prime \prime} z^{\prime \prime}$, having its interior in $K$, which does not intersect $\bar{V}$. We may find a point $c$ in $K$ and three simple arcs $c x^{\prime \prime}, c y^{\prime \prime}, c z^{\prime \prime}$, disjoint except for $c$, having their end points $x^{\prime \prime}, y^{\prime \prime}, z^{\prime \prime}$ in $E$, but otherwise lying in $K$. Now let $a$ be any non 2-point of $E$. Then $c$ is not biconjugate to $a$, hence there exists a 2 -set $H$ separating $C$ be- 
tween $a$ and $c$. Let $L$ be the component of $C-H$ which contains $c$. Since $H$ consists of two points, $L$ must contain one of the three arcs $c x^{\prime \prime}, c y^{\prime \prime}, c z^{\prime \prime}$, say $c x^{\prime \prime}$. Thus $x^{\prime \prime}$ is not biconjugate to $a$, which contradicts Theorem $\mathrm{I}(\mathrm{c})$. This proves (a).

(b) Evidently $r$ and $s$ are both local separating points of $C$. Neither of these points is of order one in $C$, since $C$ is cyclic. Hence (b) will be proved if we show that $r$ is not of order 2, since the same proof will hold for $s$. We first observe that $E$ contains a non 2-point distinct from both $r$ and $s$. If $(r, s)$ is a 2 -set, this is immediate. Otherwise there exists a free arc $r s$ in $C$. By Theorem $\mathrm{I}(\mathrm{k})$, $r s$ is in $E$, hence by Theorem $\mathrm{I}(\mathrm{i})$ every interior point of $r s$ is a non 2-point. Therefore, using Theorem I(d), we may assume that the non 2-point $a$ used to define $E$ is neither $r$ nor $s$. Let $z$ be any point of $K$ and define

$$
4 d=\min (\rho(a, r), \rho(z, r), \rho(r, s)) \geqq 0 .
$$

Assuming that $r$ is of order 2 in $C$, let $U(r)$ be a neighborhood of $r$ in $C$ of diameter less than $d$, having two points $y$ and $w$ as its boundary. From the definitions of $K$ and $d$ we may assume without loss of generality that $w$ lies in $K$ and $y$ in $C-\bar{K}$. Thus $(w, y)$ separates $C$ between $a$ and $r$. Since $r$ is in $E$, it follows by Theorem I(c) that there must exist a free arc wy in $C$. We have at once that either $r$ or $s$ is interior to the free arc wy. Call the one of these points satisfying this condition $t$. Now $w$ is not biconjugate to $a$, hence there exists a 2 -set $(h, k)$ separating $C$ between $w$ and $a$. Since $t$ is in $E,(h, k)$ does not separate $C$ between $a$ and $t$. Thus either $h$ or $k$ is $t$ or $(h, k)$ separates $C$ between $t$ and $w$. Hence in either case one of the two points $h, k$, say $h$, must be interior to the free arc $w y$. Thus $k$ does not lie on $w y$, and we see at once that if $p$ is any point interior to wy then $(p, k)$ is a 2 -set separating $C$ between $a$ and $w$. Taking $p$ interior to the subarc $t y$ of $w y$, we have at once that $(p, k)$ separates $C$ between $a$ and $t$, which is impossible since $t$ lies in $E$. The proof of (b) is thus complete.

(c) We postpone the proof of this part of Theorem II until after the proof of Theorem VI(a) in order to establish some results which we shall need in its demonstration. To this end let $R$ be the collection of all 2-points of $C ; L$ the collection of all non im kleinem cut points* of $C$; and $W$ the set of all ramification points of $C$.

LEмMа 2. Every nondegenerate subcontinuum $G$ of $R$ contains a free arc of $C$.

Proof. Since $G$ consists entirely of local separating points of $C$ it follows $\dagger$

* For definitions of the new terms used see G. T. Whyburn, Mathematische Annalen, vol. 102 (1930), pp. 313-336. We shall use Theorem 10, p. 320, of this paper and refer to it as Theorem B: Let $T$ be any arc of $C$. Then if $W$ is dense on $T, L T$ has at least the power of the continuum.

† See G. T. Whyburn, Bulletin of the American Mathematical Society, vol. 39 (1933), p. 97. 
that $G$ is a locally connected continuum. Let $T$ be any simple arc in $G$. It follows ${ }^{*}$ that $T$ contains at most a countable number of points of order different from 2.

Since $T$ consists entirely of local separating points of $C$, it follows by Theorem $\mathrm{C}$ that every point of $T$ is an im kleinem cut point. Thus $L T=0$. Therefore, $W$ is not dense on $T$, by virtue of Theorem $\mathrm{B}$. Hence $T$ contains a subarc $T^{\prime}$ which is a free arc of $C$. Since $T^{\prime}$ is contained in $G$, the proof is complete.

THEOREM III. (a) Every continuum of convergence $K$ of $C$ is contained in some true secondary element $E$ of $C$. Moreover, every such $K$ contains a non 2-point of $C$.

(b) If $\left(K_{i}\right)$ be an infinite sequence of disjoint subcontinua of $C$ having a nondegenerate limit inferior $L$, then there exists a true secondary element $G$ of $C$ which contains $L$, and is such that $L=\lim \inf K_{i} G$.

Proof. (a) Since $K$ is a continuum of convergence of $C$, no two points of $K$ can be separated in $C$ by a finite set. Thus every two points of $K$ are biconjugate and, in particular, $K$ contains no free arc. Hence the proof is complete if we show that $K$ contains at least one non 2-point of $C$. This fact follows at once from Lemma 2.

(b) $\dagger$ It follows from the disjointness of the $K_{i}$ that $L$ is a continuum of convergence of $C$, hence by (a) there exists a true secondary element $G$ of $C$ which contains $L$. Let $L^{\prime}$ be the limit inferior of $\left(K_{i} G\right)$. We have at once that $L^{\prime}$ is contained in $L$ and need only show the reverse.

To this end let $q$ be any point of $L$, and $\left(q_{i}\right)$ any sequence of points converging to $q$ such that $q_{i}$ lies in $K_{i}$ for each $i$. If $q$ is not in $L^{\prime}$, we may assume without loss of generality that $q_{i}$ lies in $K_{i}-G$ for every $i$. Let $S_{i}$ be the component of $C-G$ containing $q_{i}$. By definition $q$ lies in $F\left(\sum S_{i}\right)$, and $\ddagger$

$$
F\left(\sum S_{i}\right) \subset \overline{\sum F\left(S_{\imath}\right)} \text {. }
$$

Assume for the moment that there exists an $S_{i}$, call it $S$, which contains infinitely many of the sets $K_{i}$. It follows at once that every point of $L$ is a boundary point of $S$, contrary to Theorem II(a).

We shall show in Theorem IV that for any positive number $d$ there can

* See G. T. Whyburn, Monatshefte für Mathematik und Physik, vol. 36, pp. 305-314. We shall use Theorem 9 of this paper and call it Theorem A: All save possibly a countable number of the local separating points of a continuum $M$ are points of order 2 of $M$. We shall also refer to Theorem 8 of this paper as Theorem C: The properties of being an im kleinem cut point and of being a local separating point are equivalent for all points of a continuum $M$ at which $M$ is locally connected.

† See Kuratowski and Whyburn, loc. cit. The present proof is a modification of the proof of $(6,7)$ of this paper.

‡ See Hausdorff, Mengenlehre, Berlin, 1927, p. 155. 
exist at most a finite number of $S_{i}$ having diameter greater than $d$. From this result and the previous paragraph it follows easily that we may pick a subsequence of the $S_{i}$, which we may assume is the whole sequence, consisting of distinct elements, and then a subsequence of the $K_{i}$, which we take as the whole sequence, such that each $K_{i}$ contains a point $r_{i}$ not in $S_{i}$ and a point $q_{i}$ in $S_{i}$. It follows at once that for every $i$ the set $K_{i}$ contains a point $p_{i}$ of $F\left(S_{i}\right)$. Hence $p_{i}$ converges to $q$ since $q_{i}$ converges to $q$. But $p_{i}$ lies in $G \cdot K_{i}$, for each $i$; thus $q$ belongs to $L^{\prime}$, and the proof will be complete when we establish Theorem IV.

THEOREM IV. If $E$ be a true secondary element, then there exists for every positive number $d$ at most a finite number of distinct components of $C-E$ having diameter greater than $d$.

Proof. Otherwise there exists an infinite sequence $\left(S_{i}\right)$ of distinct components of $C-E$ each of diameter greater than $d$. We lose no generality in assuming that $\left(S_{i}\right)$ is a convergent sequence to the limit set $L$. From the fact that $E$ is closed (Theorem $\mathrm{I}(\mathrm{b})$ ) we deduce immediately that $L$ lies in $E$. Since $L$ is a connected set of diameter at least $d$, let $a, b, c$ be any three distinct points of $L$. Choose $U, V, W$ as regions in $C$ having disjoint closures and containing $a, b, c$ respectively. Let $S_{k}$ be an $S_{i}$ intersecting all three of the regions $U, V, W$. It then follows easily that $S_{k}$ has at least three boundary points, contrary to Theorem II(a).

This completes the proof of Theorems III and IV.

TheOREM V. If $E$ be a true secondary element and $N$ a component of $C-E$, then for every true secondary element $F$ we have either $F N=0$ or $\bar{N} \supset F$.

Proof. Let $a$ and $b$ be non 2-points defining $E$ and $F$ respectively. Then there exists a 2 -set $(h, k)$ which separates $C$ between these two points. It follows easily that $C-(h, k)$ has exactly $n$ components $A_{i},(i=1,2, \cdots, n)$, for some finite $n$. We lose no generality in assuming that $b$ lies in $A_{1}$ and $a$ in $A_{2}$. It follows at once that $F$ is contained in $\bar{A}_{1}$, and $E$ is contained in $\bar{A}_{2}$. But $C-E$ contains $C-\bar{A}_{2}=A_{1}+\sum_{3}^{n} A_{i}$; hence if $N$ is the component of $C-E$ containing $b$ we have that $\bar{N}$ contains $\bar{A}_{1}$ which contains $F$. Thus $\bar{N} \supset F$. Evidently, $F M=0$ for every other component $M$ of $C-E$. This completes the proof of Theorem $\mathrm{V}$.

LEMma 3. If $E$ be a true secondary element, and $K$ a component of $C-E$ with boundary points $r$ and $s$, then $\bar{K}$ contains at most one true secondary element $F$ of $C$ containing $r+s$.

Proof. Assume that there exist two such true secondary elements $F$ and $G$ of $C$. By Theorem I(f), $(r, s)$ is a 2-set; hence both $r$ and $s$ are 2-points. Let $a$ 
and $b$ be non 2-points defining $F$ and $G$, respectively, and let $a x b$ be a simple $\operatorname{arc}$ in $K$. Let $w$ be the last point of $a x b$ in $F$ and $z$ the first point of $a x b$ following $w$ which is in $G$. By Theorem I(f), $w$ and $z$ are distinct points; hence the arc $w z$ is nondegenerate. If $M$ be the component of $C-G$ containing $a$, then $\bar{M}$ contains $F$ by Theorem V. It follows that $M$ has the three boundary points $r, z, s$, contrary to Theorem II(a). The proof is thus complete.

Theorem VI. (a) For every positive number $d$ there exists at most a finite number of true secondary elements $E$ having components of diameter greater than $d$.

(b) C contains at most a countable number of distinct true secondary elements.

Proof. Contrary to the theorem, let $\left(E_{i}\right)$ be an infinite sequence of distinct true secondary elements having components of diameter greater than $d$. The proof now splits into two cases:

CASE (i). For each $i$ the set $E_{i}$ intersects at most a finite number of the sets $E_{j}$.

In this case there is an infinite subsequence of the $E_{i}$ no two of which have a point in common, and we may assume this is the entire set $E_{i}$. Let $e_{i}$ be a component of $E_{i}$ of diameter greater than $d$. We may assume that the sequence $\left(e_{i}\right)$ converges to a limit set $e$ of diameter at least $d$. From Theorem III it follows that there exists a true secondary element $F$ of $C$ containing $e$. We may assume $F$ is distinct from all the $E_{i}$. Using Theorems IV and V, we may assume that there exists a component $N$ of $C-F$ such that $\bar{N}$ contains $E_{i}$ for every $i$. Using Theorem II(a) we may assume that all the $E_{i}$ are disjoint with $F$. Hence all the $\left(e_{i}\right)$ are contained in $N$, a single component of $C-F$ which gives an easy contradiction to Theorem $\mathrm{II}(\mathrm{a})$ since $C$ is a continuous curve. Thus the theorem is proven for Case (i).

CASE (ii). There exists an $E_{i}$ intersecting infinitely many $E_{j}$.

We may assume in this case that $E_{1}$ intersects all the remaining $E_{i}$, and, by Theorems IV and $\mathrm{V}$, that there exists a component $N$ of $C-E_{1}$ such that $\bar{N}$ contains all the remaining $E_{i}$. Using Theorem II(a), let $r$ and $s$ be the boundary points of $N$. By Lemma 3 we may assume that every $E_{i}$ contains $r$ and no $E_{i}$, other than $E_{1}$, contains $s$. Continuing, this process of taking subsequences indefinitely we may assume without loss of generality that for $i$ not $j$ we have $E_{i} E_{j}=r$. Let $q$ lie in $E_{1}-(r, s)$ and define $5 m=\rho(q, \bar{N}), 5 n=d$ and $k=\min (m, n)$. If we define $d^{\prime}=3 k$, we have at once that $d^{\prime}$ is less than $d$.

Let $e_{i}$ be a component of $E_{i}$ having diameter greater than $d^{\prime}$. It follows easily that there exists a point $p_{i}$ in $e_{i}$, such that $\rho\left(p_{i}, r\right)$ is greater than $k$. Let $V_{i}$ and $U$, respectively, be the spherical $k$-neighborhoods of $p_{i}$ and $q$; and 
define $G_{i}$ as the sum of these neighborhoods. We shall assume, henceforth, that $i$ is not one. We note in passing that $\bar{U}$ and $E_{i}$ are disjoint for all such $i$. We now know that $e_{i}$ is a continuum and $G_{i}$ is an open set intersecting both $e_{i}$ and $C-e_{i}$. It follows, therefore, by a well known theorem* that the component $K_{i}$ of $\bar{G}_{i} e_{i}$ which contains $p_{i}$ contains at least one point of $F\left(G_{i}\right)$, and hence at least one point of $F\left(V_{i}\right)$. Thus $K_{i}$ is a continuum of diameter at least $k$ which does not contain $r$. The $K_{i}$, being subsets of the corresponding $E_{i}$, are disjoint by pairs. The proof given for (i) now holds exactly if we substitute the sets $K_{i}$ for the sets $e_{i}$ used in that proof, and $k$ for $d$. This leads to the same contradiction and completes the proof of (a).

We postpone the proof of (b) until after the development of some special theory of degenerate true secondary elements. Meanwhile we give the following proof.

Proof of Theorem II(c). Assuming the theorem false we first prove

(i) $K$ contains a continuum of convergence of itself. Furthermore, every simple closed curve $J$ containing $r, s$ and a point of $K$ intersects some continuum of convergence of $K$ in at least two points. Moreover, the true secondary element $F$ of $C$ which contains this continuum of convergence is contained entirely in $K$.

Proof. Let $J$ be any such simple closed curve and let $A$ be any simple arc of $J$ joining $r$ and $s$ in $\bar{K}$. Since $(r, s)$ is not a 2-set, but does separate $C$, it follows that there exists a free $\operatorname{arc} B=r x s$ in $C$, and $B$ is in $E$ by Theorem $\mathrm{I}(\mathrm{k})$. Thus if we define $J^{\prime}=A+B$, it follows that $J^{\prime}$ is a simple closed curve such that $J^{\prime} K$ is contained in $J K$. Hence it suffices to prove the theorem using $J^{\prime}$ in place of $J$.

From our hypothesis and Theorem I(i) it follows that every point of $B$ is a non 2-point, hence no point of $K$ is biconjugate to any point of $B$. Let $x$ be an interior point of $A$; then there exists a 2 -set $\left(h_{1}, k_{1}\right)$ separating $r$ from $x$ in $C$. It follows at once that both $h_{1}$ and $K_{1}$ are interior to $A$ and that we may assume that $h_{1}$ lies between $r$ and $x$ and $k_{1}$ between $x$ and $s$ on this arc.

Now $\left(r, k_{1}\right)$ does not separate $C$. If it did, since $r$ is a non 2-point, we could find a free arc $r k_{1}$ in $C$. It follows easily that this gives a theta curve in $C$ having $r$ and $k_{1}$ as vertices. This is impossible since $k_{1}$ is not biconjugate to $r$.

Since $\left(r, k_{1}\right)$ does not separate $C$, there exists a simple arc $W$ joining $x$ to $a$ (an interior point of $B$ ) but containing neither $r$ nor $k_{1}$. Since $\left(h_{1}, k_{1}\right)$ separates $C$ between $x$ and $s$, and since no interior point of $A$ is biconjugate to $s$, we may find a simple arc $T_{1}$ in $K$ spanning $J^{\prime}$ and having its end points $a_{1}, b_{1}$ interior to the subarcs $r h_{1}$ and $s k_{1}$ of $A$, respectively, except that $a_{1}$ may possibly be $h_{1}$.

* See Hausdorff, loc. cit., p. 161. 
Now $a_{1}$ is not biconjugate to $r$; hence there exists a 2 -set $\left(h_{2}, k_{2}\right)$ separating $a_{1}$ from $r$ in $C$, and we may assume that $h_{2}$ is interior to $r a_{1}$ and $k_{2}$ is on the half open $\left.\operatorname{arc} s b_{1}\right\rangle$. Using the argument of the preceding paragraphs, we may find a simple arc $T_{2}$ in $K$ spanning $J^{\prime}$ between $a_{2}$ and $b_{2}$. These points will lie respectively in the half open arc $\left\langle r h_{2}\right.$ and the open arc $\left\langle s k_{2}\right\rangle$. It follows at once that $\left(h_{2}, k_{2}\right)$ separates $C$ between every point interior to $T_{1}$ and every interior point of $T_{2}$ whence an easy consequence is that $T_{1}, T_{2}$ are disjoint.

In the same manner we may find an infinite sequence $\left(T_{i}\right)$ of disjoint simple arcs in $K$ spanning $J^{\prime}$ each between a point of $r h_{1}$ and a point of $s k_{1}$; hence all of the $T_{i}$ are of diameter as least as great as the distance between these disjoint closed sets. Thus we may assume that the $T_{i}$ converge to a nondegenerate limit set $T$. Hence by Theorems III(a), I(f), V, the fact that both $r$ and $s$ are non 2-points, and the following assertion (which follows easily from Theorems II(a) and III(b)), there must exist a true secondary element $F$ of $C$ which contains $T$ and is contained in $K$.

ASSERTION. If E be a true secondary element, then there exists no convergent infinite sequence of disjoint subcontinua of $C-E$ each member of which is of diameter greater than some preassigned $d$ such that the limit of this sequence is in $E$.

From the compactness of $r h_{1}$ and $s k_{1}$ we have at once that $T$ must intersect the interiors of both of these arcs. This completes the proof of (i).

We continue to use the simple closed curve $J^{\prime}$ defined in proving (i). We see by that Theorem that $K$ contains a continuum of convergence $t_{1}$ of itself which is contained in a true secondary element $E_{1}$ of $C$. Moreover, by (i), $E_{1}$ is contained in $K$ and has at least two points in $J^{\prime}$. It follows at once that these points must both be interior to $A$. Let $m_{1}, n_{1}$ be respectively the first and last points of the closed set $E_{1}$ in $A$. Define

$$
d=\min \left(\operatorname{diam} t_{1}, \rho\left(r m_{1}, s n_{1}\right)\right) .
$$

We shall now prove the following assertion:

(ii) $\left(r, m_{1}\right)$ does not separate $C$.

Proof. Otherwise, since $r$ is a non 2-point, there exists a free $\operatorname{arc} r m_{1}$ in $C$. By Theorem II(b), $s$ is not interior to this free arc; hence $\left\langle r m_{1}\right.$ is contained in $K$. Let $z$ be any point of $\left\langle r m_{1}\right\rangle$. Then $(s, z)$ separates $C$ between $r$ and $m_{1}$. Otherwise there exists a simple arc rwm in $C-(s, z)$, and it follows easily that $\left\langle r w m_{1}\right.$ lies in $K$. Since $r w m_{1}$ does not contain $z$, it must be disjoint with the interior of the free arc $r m_{1}$. It is easily established from these facts that $K$ contains a point $w$ which can be joined to $r$ by three independent $\operatorname{arcs}$ of $C$. This is impossible since no point of $K$ is biconjugate to $r$. 
Therefore, $(s, z)$ separates $C$. Thus, since $s$ is a non 2-point, there must exist a free arc $s x z$ in $C$. This free arc cannot contain $r$ by Theorem $\mathrm{II}(\mathrm{b})$, hence it must contain $m_{1}$. But the half open arc rym $\left.m_{1}\right\rangle$ is contained in a single component $N$ of $C-E_{1}$, by definition of $m_{1}$. This gives a contradiction to Theorem II(b), and thus establishes (ii).

It is immediate that there exists a component $N$ of $C-E_{1}$ such that $F(N)=\left(m_{1}, n_{1}\right)$; thus $\left(m_{1}, n_{1}\right)$ separates $C$. Therefore, by (ii), there exists a simple arc $x y$ in $K$ spanning $J^{\prime}$, where $x$ is in $\left\langle r m_{1}\right\rangle$ and $y$ in $s n_{1}$. Thus $x$ is biconjugate to $y$, whence $y$ is not $s$.

Using the notation of (i) we find a 2 -set $\left(h_{1}, k_{1}\right)$ separating $r$ from $x$ in $C$, where $h_{1}$ is in $\langle r x\rangle$ and $k_{1}$ in $\left\langle s n_{1}\right\rangle$. It follows at once that $\left(h_{1}, k_{1}\right)$ separates $C$ between $r$ and $n_{1}$. Continuing as in (i) we obtain a continuum of convergence $t_{2}$ of $K$ intersecting both $s k_{1}$ and $\left\langle r h_{1}\right\rangle$. We let $E_{2}$ be the true secondary element of $C$ containing $t_{2}$, and note that $E_{2}$ is contained in $K$ and is of diameter at least $d$. Also, $E_{1}$ and $E_{2}$ are distinct. Repeating this process indefinitely gives an infinite sequence $\left(t_{i}\right)$ of continua of convergence of $K$, each lying in a different true secondary element of $C$ and each of diameter at least $d$. This contradicts Theorem VI(a) and completes the proof of Theorem II(c).

THEOREM VII. If $E$ be a degenerate true secondary element, then every two points $p$ and $q$ of $E$ are vertices of a theta curve in $C$.

Proof. By a theorem of N. E. Rutt* our theorem is equivalent to showing that no pair of points $(h, k)$ separates $p$ and $q$ in $C$. Assuming it false, let $J$ be any simple closed curve in $C$ containing $p$ and $q$; then $J$ contains $h$ and $k$. By Theorem $\mathrm{I}(\mathrm{c})$ there exists a free $\operatorname{arc} h x k$ in $C$.

CASE (i). $p$ is a non 2-point.

If $h x k$ contains $p$, then by Theorem $\mathrm{I}(\mathrm{j})$ the free $\operatorname{arc} h x k$ is contained in $E$, which is impossible since $E$ is degenerate. If $h x k$ contains $q$, then, since $q$ is biconjugate to $p$, every interior point of $h x k$ is biconjugate to $p$, and we are led to the same contradiction through Theorem $\mathrm{I}(\mathrm{d})$.

Thus $h x k$ spans $J$, whence $h$ is biconjugate to $k$, and by Theorem $\mathrm{I}(\mathrm{h})$, every interior point $c$ of $h x k$ is a non 2-point. Thus $h x k$ lies in a nondegenerate true secondary element $F$ of $C$. By Theorem $\mathrm{I}(\mathrm{f}), c$ is not in $E$; hence $c$ is not biconjugate to $p$. This yields an easy contradiction to the fact that $p$ and $q$ are biconjugate points, since any 2-set which separates $c$ from $p$ in $C$ will also separate $p$ from $q$. Thus Case (i) is established.

* See N. E. Rutt, American Journal of Mathematics, vol. 51 (1929), pp. 217-246, where the theorem is established for the plane. (Note in particular Theorem 4, p. 244, of this paper.) For the proof of the theorem for more general spaces see G. Noebeling, Fundamenta Mathematicae, vol. 18 (1932), p. 23-38. 
CASE (ii). Both $p$ and $q$ are 2-points.

There exists a point $z$ such that $(p, z)$ is a 2 -set. By Theorem $\mathrm{I}(\mathrm{c})$ there exists a component $K$ of $C-(p, q)$ such that $\bar{K}$ contains $E$; hence $K$ contains a non 2-point $a$ of $E$. Let $b$ be any point of $C-\bar{K}$, and $J$ any simple closed curve in $C$ containing $a$ and $b$. Thus $J$ contains both $p$ and $q$. The half open arc $\langle p b$ of $J$ is contained in $C-\bar{K}$ and hence in $C-E$. Thus $p$ is a boundary point of some component of $C-E$. Hence by Theorem II(b), $p$, and similarly $q$, is a local separating point of $C$ of order greater than 2. Thus $h x k$ spans $J$; hence $h$ is biconjugate to $k$, and, by Theorem $\mathrm{I}(\mathrm{h})$, every interior point of $h x k$ is a non 2-point. Let $c$ be any interior point of $h x k$ and $F$ the true secondary element of $C$ containing $c$. Since $p$ is biconjugate to $q$, it follows easily that both $p$ and $q$ are biconjugate to $c$, hence, Theorem I(f), $E F=(p, q)$ and $(p, q)$ is a 2 -set. $F$ is also nondegenerate, when considered as a true secondary element. If $b$ be a non 2-point defining $E$, it follows at once that $b$ is not on the free arc $h x k$. Thus $(h, k)$ separates $C$ between $b$ and either $p$ or $q$, say $p$. Let $J^{\prime}$ be a simple closed curve in $C$ containing $p$ and $b$; then $J^{\prime}$ contains $h$ and $k$ and is spanned by the simple arc $h x k$. It follows that $b$ is biconjugate to $c$, since $p$ is biconjugate to $b$. Hence $E F$ contains the three distinct points $p, q, b$ contradicting Theorem $\mathrm{I}(\mathrm{f})$ and proving the theorem.

In the first paragraph of the proof of Theorem VII we established the following theorem.

THEOREM VIII. Every 2-point pof $C$ which is contained in a true secondary element $E$ of $C$ is a local separating point of $C$ of order greater than 2 and also a boundary point of some component of $C-E$.

THEOREM IX. If $E$ be a true secondary element containing no free arc, and $K$ is any component of $C-E$, then $F(K)$ is a 2-set.

The proof is immediate from Theorems I(k), II(a), and II(b).

THEOREM $\mathrm{X}$. If $E$ be a degenerate true secondary element, then $E$ contains infinitely many 2-sets and $C-E$ contains an infinite sequence of components whose closures are disjoint by pairs.

Proof. Let $a$ be any non 2-point of $E$ and $b$ an arbitrary point of $E-a$. By Theorem VII there exists a theta curve $H=\sum_{1}^{3} a x_{i} b$ in $C$ having $a$ and $b$ as vertices. Using Theorem $\mathrm{I}(\mathrm{k})$ and the fact that $a$ is a non 2-point, we have at once that $C-(a, b)$ is connected. It follows that there is an arc $h_{1} k_{1}$ spanning $H$ between $a x_{1} b$ and one of its other two arcs. We lose no generality if we assume that $h_{1}$ lies in $\left\langle a x_{1} b\right\rangle$ and $k_{1}$ in $\left\langle a x_{2} b\right\rangle$. It is immediate that both $h_{1}$ and $k_{1}$ are in $E$ since both of these points are biconjugate to $a$. 
We have thus shown that for any point $b$ in $E-a$ and any theta curve $H$ in $C$ having $a$ and $b$ as vertices, there exists a point $q$ ( $h_{1}$ above) interior to $a x_{1} b$ such that $q$ and $a$ are vertices of a theta curve in $C$.

Using $h_{1}$ as $b$ we may find an $h_{2}$ in $\left\langle a x_{1} h_{1}\right\rangle$ such that $h_{2}$ is in $E$ and $h_{2}$ and $a$ are vertices of a theta curve in $C$. Continuing this process indefinitely we may construct an infinite sequence of points $\left(h_{i}\right)$ such that for every $i$ we have

(a) $h_{i}$ is in $E$;

(b) $a$ precedes $h_{i+1}$ precedes $h_{i}$ precedes $b$ on $a x_{1} b$;

(c) $h_{i}$ and $a$ are vertices of a theta curve in $C$.

We may assume that the sequence $\left(h_{i}\right)$ converges to a point $h$. We have that $a$ lies on $a x_{1} b$ and precedes all the $h_{i}$ on this arc.

Since $E$ is degenerate there exists for each $n$ a point $p_{n}$ in the subarc $\left\langle h_{2 n} x h_{2 n-1}\right\rangle$ of $a x_{1} b$ which is not in $E$. Hence, (Theorem I(b)), let $w_{n}, z_{n}$ be respectively the last point of $E$ preceding and the first point of $E$ following $p_{n}$ on $a x_{1} b$. We have at once that both of these points are contained in the closed arc $h_{2 n} x_{1} h_{2 n-1}$; hence if $K$ is the component of $C-E$ containing $p_{n}$ it follows from Theorem II(a) that $F\left(K_{n}\right)=\left(w_{n}, z_{n}\right)$.

We have at once that the infinite sequence of components $\left(K_{2 i-1}\right)$ of $C-E$ are mutually separated by pairs. The remainder of the theorem follows at once from Theorem IX.

Proof of Theorem VI(b). By virtue of Theorem VI(a) it suffices to prove that $C$ contains but a countable number of degenerate true secondary elements. Assume the theorem false and let $(E)$ be any uncountable collection of degenerate true secondary elements of $C$. Let

$$
p_{1}, p_{2}, p_{3}, \cdots
$$

be the set of all local separating points of $C$ contained in at least one of the sets $(E)$. This set is countable by Theorem A. Using Theorems VIII and X, we see that every set $E$ contains infinitely many distinct points of (1). It follows that some point of (1), say $p_{1}$, must be contained in an uncountable subcollection of the $(E)$; hence we lose no generality in assuming that it is contained in all the $E$. Let $E_{1}$ be any one of these sets. From Theorems IV and V we lose no generality in assuming that there exists a component $K_{1}$ of $C-E_{1}$ such that $\bar{K}_{1}$ contains all the sets $(E)$ except $E_{1}$. Using Theorem II(a) and Lemma 3, we may let $F\left(K_{1}\right)=\left(p_{1}, s_{1}\right)$ and assume that $s_{1}$ lies in none of the sets $E$ other than $E_{1}$. From Theorems VIII and X every $E$ contains a 2-point $q$ distinct from $p_{1}$. From this it follows easily that we may assume $p_{2}$ lies in uncountably many $E$; hence uncountably many $E$ 's contain both $p_{1}$ and $p_{2}$. This gives an easy contradiction to Lemma 3 using Theorem IV. The proof of Theorem VI(b) is thus completed. 
Theorem XI. Every isolated point $q$ of a true secondary element $E$ is a 2point.

Proof. Let $a$ be any non 2-point of $E$ and $a k q$ a simple arc in $C$. Let $k$ be the last point of $E$ preceding $q$ on $a k q$. It follows at once from Theorem II(a) that there exists a component $K$ of $C-E$ such that $F(K)=(k, q)$. The theorem is then immediate by Theorem $\mathrm{I}(\mathrm{k})$.

Notation. For any set $M$ let $L(M)$ denote the number of components of $M$, if $M \neq 0$, and $L(M)=1$ if $M=0$.

THEOREM XII.* Let $F$ be a closed subset of a compact continuum $S$ such that each component of $S-F$ has a most $n$ limit points in $F$. Then for any continuum $N$ of $S$, we have

$$
L(N F) \leqq n L(S-F) .
$$

Proof. We have only to consider the case where $L(S-F)$ is finite and $N \nsubseteq F$, as the inequality is obvious in the other cases. Let $P$ be the set of all limit points in $F$ of components of $S-F$. Then the number of points in $P$ is less than $n L(S-F)$. Let $q$ be a point of $N F-P$. By a theorem of Hausdorff the component of $N$ containing $q$ and lying in the open set $U=S-\overline{S-F}$ has a limit point on $F(U)$. But this component belongs to $N F-P$ and $F(U) \subset P$. Thus each component of $N F$ contains a point of $P$ which proves the theorem.

Corollary. For any true secondary element $E$ of $C$ and any continuum $N$ in $C$ we have

$$
L(N E) \leqq 2 L(C-E) .
$$

TheOREM XIII. If $Z(E)$ is finite, and $K$ is a continuum of convergence of $C$ which is contained in $E$, then $K$ contains a continuum of convergence of $E$.

Proof. Let $Z(E)=h$, and let $d$ be so chosen that the diameter of $K$ is $4 h d$. Since $K$ is a continuum of convergence of $C$, there exists an infinite sequence $\left(K_{i}\right)$ of disjoint subcontinua of $C$ converging to $K$. By Theorem III(b) the sets $K_{i} E$ will also converge to $K$. We thus lose no generality if we assume that the diameter of $K_{i} E$ is greater than $3 h d$ for all $i$. Since $K$ is a continuum of diameter $4 h d$, there will exist $3 h$ points $p_{i},(i=1,2, \cdots, 3 h)$, in $K$ such that $\min \rho\left(p_{i}, p_{j}\right)=d, \quad(i \neq j)$. Construct the neighborhoods $V_{i}\left(p_{i}\right)$, $(i=1,2, \cdots, 3 h)$, in $C$ having disjoint closures. For $i$ sufficiently large $K_{i} E$ will intersect all of these neighborhoods. By the corollary to Theorem XII, $K_{i} E$ has at most $2 h$ components for every $i$. It follows that for every sufficiently large $i$ some component $k_{i}$ of $K_{i} E$ must intersect at least two of the neighborhoods $V_{i}\left(p_{i}\right)$. Using this fact and the information that the $V_{i}\left(p_{i}\right)$

* This theorem and its proof were suggested by the referee. 
have disjoint closures, the required continuum of convergence of $E$ is easily constructed.

\section{Part II. Secondary EXTENSIBILITY AND REDUCIBILITy}

Definition. Let $P$ be a property of point sets. Then $P$ will be said to be secondarily extensible provided $C$ has property $P$ whenever every component of each true secondary element of $C$ has property $P$. The property $P$ will be said to be secondarily reducible provided the assertion $C$ has property $P$ implies that every component of each true secondary element $E$ of $C$ has property $P$.

THEOREM XIV. Let $C$ have the property that for each true secondary element $E$ of $C, L(C-E)$ is finite. Let $P$ be any one of the following properties: (1) being hereditarily locally connected; (2) being a regular curve; (3) being a rational curve. Then $P$ is secondarily extensible and reducible.

Proof. The proof that each of these properties is secondarily reducible follows at once from the fact that if $C$ has property $P$ then every subcontinuum of $C$ necessarily has property $P$.

(1) is secondarily extensible.

Otherwise there exists a continuum of convergence $K$ of $C$. Using Theorems III(a) and XIII we obtain at once a continuum of convergence of some component of a true secondary element $E$ of $C$. This contradiction establishes our assertion.

(2) and (3) are secondarily extensible.

Assume that $C$ is not a regular (rational) curve. Then, by a theorem of Menger, ${ }^{*} C$ contains a subcontinuum $N$ composed entirely of irregular (irrational) points of $C$. Using two theorems of G. T. Whyburn, $\dagger$ we see that the non-local separating points of $C$ are dense on $N$. Let $a$ be any non-local separating point of $C$ contained in $N$. Then $a$ is a non 2-point of $C$. Since $a$ is of order greater than 2, it is not a 2 -end point of $C$ and hence defines a true secondary element $E$ of $C$. Since $Z(E)$ is finite and $a$ is not a local separating point of $C$, it follows that $a$ is not a limit point of $C-E$. Hence by Theorems $\mathrm{XI}$ and XII we see that $a$ lies in a nondegenerate component $K$ of $N E$. Let $M$ be the component of $E$ containing $K$. From the Corollary to Theorem XII we have that $a$ is not a limit point of $C-M$, hence the order of $a$ in $M$ is the same as the order of $a$ in $C$. Therefore, $a$ is an irregular (irrational) point of $M$. Thus $M$ is not a regular (rational) curve. This contradiction establishes (2) and (3).

* See K. Menger, Kurventheorie, Leipzig, 1932, pp. 127, 133.

$\dagger$ See Bulletin of the American Mathematical Society, vol. 35 (1929), p. 102, Theorems 13 and C. 
THEOREM XV. The property of being a regular curve is not secondarily extensible.*

Proof. The proof will be by an example constructed in the Euclidean plane $P$. Let $A B D$ be an equilateral triangle in $P$ with base $A D$. Let $E$ be the midpoint of $A D$ and join $E$ to the midpoints of $A B$ and $B D$ by line segments $E F$ and $E G$ respectively. There will then be two triangles (namely $A F E$ and $E G D$ ) having their bases on $A D$. Repeat the above construction on each of these triangles, and continue the process indefinitely. Call the resulting configuration $K$. Let $K^{\prime}$ be the reflection of $K$ in $A D$ with $B^{\prime}$ the point corresponding to $B$ under the reflection. Through each point of $A B^{\prime}$ having order three construct a segment parallel to $A D$ and extended until it meets the segment $B^{\prime} D$. Denote by $H$ the set $K^{\prime}$ modified by the addition of these intervals. Let $C^{\prime}$ consist of $H, K$ and a free arc $B B^{\prime}$ formed by two segments and spanning the point set $H+K$. Let $t$ be any free arc of $H+B B^{\prime}$ and $p$ any interior point of $t$. With $p$ as center and sufficiently small radius we may draw a circle disjoint with $\overline{C^{\prime}-t}$, but lying in the plane of $C^{\prime}$. Let $C$ be the set $C^{\prime}$ modified by the addition of such a circle to every free arc of $H+B B^{\prime}$. Then the secondary elements of $C$ may be classified as follows: (1) one disconnected nondegenerate true secondary element $G$ consisting of $K$ and all points of $H+B B^{\prime}$ of order greater than two; (2) a countable number of connected nondegenerate true secondary elements, each consisting of a theta curve composed of a circle and a proper subinterval of some free arc of $H+B B^{\prime}$; (3) a countable number of secondary elements of type III each consisting of the closure of a component of the complement in $C$ of all secondary elements mentioned in (1) and (2).

It will be noted that although no secondary element of $C$ contains an irregular point of itself, the set $C$ is not even hereditarily locally connected.

\section{PART III. EXAMPLES}

THEOREM XVI. There exist true secondary elements $E$ having each of the following properties:

(a) $E$ is connected and locally connected;

(b) $E$ is connected but not locally connected;

(c) $E$ is locally connected but not connected;

(d) $E$ is countable;

(e) $E$ is uncountable;

(f) $E$ is the closure of a free arc;

(g) E is cyclic;

* Cf Theorem XIV. 
(h) E has exactly one end point of itself;

(i) $E$ is neither connected nor locally connected.

Proof. (a), (e), (g): Let $C$ be a 2-cell; then $E=C$.

For the remaining examples we define a lumped free arc of $C$ to be on open subset $A$ of $C$ which contains $B$, where $A$ is an open $\operatorname{arc}, B$ is a 2-cell, and $A \cdot B$ is an arc (closed).

(c), (e): Let $C$ consist of two disjoint 2-cells $H$ and $K$ joined by three disjoint lumped free arcs; then $E=H+K$.

(e), (i): The example shall be constructed in the Cartesian plane. Let $C_{i}$ be a circle and its interior having the center $\left(2^{-i}, 0\right)$ and the radius $1 / 2^{i+2}$. It follows easily that all the $C_{i}$ are disjoint by pairs. Let $p_{i}, q_{i}$ be respectively the points of $C_{i}$ having the maximum and minimum ordinate. For each $i$ let $A_{i}$ be the line segment joining $p_{i}$ to $p_{i+1}$, and $B_{i}$ the line segment joining $q_{i}$ to $q_{i+1}$. Let $T$ denote the closed interval of the $x$ axis from $(0,0)$ to $(1 / 2,0)$, and let $D$ consist of the sum of all the sets $A_{i}, B_{i}, C_{i}, T$. In $D$ replace each free arc by a lumped free arc and denote the resulting configuration by $C$. Let $E$ denote the subset of $C$ consisting of the origin and all the sets $C_{i}$. Then $E$ is a true secondary element of $C$ fulfilling the conditions (e) and (i).

(e), (f): Let $C$ consist of a right triangle plus the three segments parallel to the hypotenuse and joining the points dividing its legs into four equal parts. Then $E$ will be the middle one of these segments.

(d), (i): Let $D^{\prime}$ consist of an equilateral triangle $p q r$, having $p$ at the origin and the side $p q$ along the positive $x$ axis, plus an infinite sequence of disjoint free arcs spanning $p q r$ between points on $p q$ and $p r$ and converging to the point $p$. Let $D^{\prime \prime}$ be the reflection of $D^{\prime}$ in the $y$ axis, where $q^{\prime}$ is the reflection of $q$ and $r^{\prime}$ is the reflection of $r$. Define $D$ as the sum of $D^{\prime}$ and $D^{\prime \prime}$ plus two disjoint spanning arcs of this set, one between $q^{\prime}$ and $q$, the other between $r^{\prime}$ and $r$. Let $C$ be the configuration $D$ in which every free arc has been replaced by a lumped free arc. Then $E$ will consist of the non 2-point $p$ plus the end points of all the lumped free arcs of $C$.

(a), (e), (h): In the Euclidean plane $P$ let $D$ be the sum of two disjoint 2-cells $A$ and $B$. Let $a, b, c$ be three distinct points of $A$ which are limit points of $P-A$ and $d, e$ two distinct points of $B$ which are limit points of $P-B$. Let $D^{\prime}$ consist of $D$ together with a spanning arc $a d$ of $D$ and a spanning lumped free $\operatorname{arc} c e$ of $D$ which is disjoint with $a d$. Let $f$ be any interior point of $a d$ and $b f$ a lumped free arc spanning $D^{\prime}$. Let $C$ consist of the entire configuration. Then $E$ will be the sum of $A$ and the free $\operatorname{arc} a f$ of $C$.

(b), (e), (g): Let $A$ be a square and its interior with center at $p$. Let $B$ be a square and its interior with side half that of $A$, having its center at $p$ 
and its sides parallel to those of $A$. Let $D=\overline{A-B}$. Then $D$ divides the plane into exactly two regions, the bounded one of which we call $R$. Thus the boundary of $R$ is a square having sides $W, X, Y, Z$ in the counterclockwise order. We define $\left(D_{i}\right)$ as an infinite sequence of disjoint 2-cells contained in $\bar{R}$, converging monotonically to $W$, and each intersecting $\bar{R}$ in exactly two simple arcs, one on $X$ and one on $Z$. (The 2-cells are most easily pictured as strips.) Add $i$ disjoint lumped free $\operatorname{arcs}$ in $R$ spanning $E$ between points of $D_{i}$ and $D_{i+1}$ and equally spaced in the region between these 2-cells. Then the resulting space $C$ is a locally connected continuum. It is easily seen that the set $E$ consisting of $D$ and all the sets $D_{i}$ is a true secondary element of $C$ satisfying (b), (e), and (g).

The University of Virginia, Charlottesville, Va. 